Article

\title{
Effects of Two Dietary Fibers as Part of Ready-to-Eat Cereal (RTEC) Breakfasts on Perceived Appetite and Gut Hormones in Overweight Women
}

\section{David W. Lafond ${ }^{1,2, \dagger, *}$, Kathryn A. Greaves ${ }^{1, \dagger}$, Kevin C. Maki ${ }^{3, \dagger}$, Heather J. Leidy ${ }^{4, \dagger}$, and Dale R. Romsos ${ }^{2, \dagger}$}

1 Kellogg Company, WKKI 2 Hamblin Ave E, Battle Creek, MI 49017, USA; E-Mail: kathy.greaves@kellogg.com

2 Department of Food Science and Human Nutrition, Michigan State University, 469 Wilson Road, East Lansing, MI 48824-1224, USA; E-Mail: dromsos@msu.edu

3 Biofortis Clinical Research/Midwest Center for Metabolic \& Cardiovascular Research, 489 Taft Avenue, Glen Ellyn, IL 60137, USA; E-Mail: kmaki@mc-mcr.com

4 Department of Nutrition \& Exercise Physiology, University of Missouri, 307 Gwynn Hall, Columbia, MO 65211, USA; E-Mail: leidyh@health.missouri.edu

$\dagger$ These authors contributed equally to this work.

* Author to whom correspondence should be addressed; E-Mail: david.lafond@kellogg.com; Tel.: +1-269-961-2817.

Received: 16 December 2014 / Accepted: 4 February 2015 / Published: 13 February 2015

\begin{abstract}
The effects of an enzyme-hydrolyzed arabinoxylan from wheat (AXOS) versus an intact arabinoxylan from flax (FLAX) added to a ready-to-eat cereal (RTEC) on the postprandial appetitive, hormonal, and metabolic responses in overweight women (BMI 25.0-29.9 $\mathrm{kg} / \mathrm{m}^{2}$ ) were evaluated. Subsequent meal energy intake was also assessed. Two randomized, double-blind, crossover design studies were completed. For trial 1, the participants consumed the following RTEC breakfast, matched for total weight and varied in energy content: low-fiber (LF, 4 g); high-fiber (HF, 15 g) as either AXOS or FLAX. For trial 2, the participants consumed LF, HF-AXOS, and HF-FLAX RTECs but also consumed another LF breakfast that was isocaloric (LF-iso) to that of the HF breakfasts. Perceived appetite and blood samples (trial 2 only) were assessed before and after breakfast. An ad libitum lunch was offered $4 \mathrm{~h}$ post-breakfast. No differences in postprandial appetite responses were observed among any breakfasts in either trial. The HF-AXOS and HF-FLAX led to increased postprandial GLP-1 and peptide YY (PYY) concentrations vs. LF-iso. No
\end{abstract}


differences were observed in lunch meal energy intake among breakfast meals in either trial. Collectively, these data suggest that $15 \mathrm{~g}$ of low molecular weight fiber added to RTECs did not affect perceived appetite or subsequent energy intake despite differences in satiety hormone signaling in overweight females.

Keywords: ready to eat cereal (RTEC); satiety; PYY; GLP-1; arabinoxylans; visual analogue scales (VAS)

\section{Introduction}

Over the last 25 years there have been many studies examining the role of increased dietary fiber intake on satiety and weight loss. A link between fiber and satiety was proposed as early as 1987 [1]. Since that time there has been a significant amount of research relating fiber to satiety [2,3]. Dosages of fiber used have varied considerably, with more consistent satiety effects above $10 \mathrm{~g}$ at a single eating occasion. However, mean daily fiber intake for adult women in the U.S. is only slightly above this threshold (about $14 \mathrm{~g} /$ day) [4] and well below the recommended $25 \mathrm{~g} /$ day [5].

It is difficult to incorporate fiber sources into existing breakfast cereal food products without changing desirable food texture and flavor characteristics. Fiber may be added either as part of an ingredient, or as an isolated fiber [6]. When fiber is added to food as part of an ingredient such as wheat bran, other components in that ingredient can contribute color, flavor, and texture, as well as additional calories to the finished food. In the case of wheat bran, these components would include starch and protein from the endosperm included in the bran mill fraction. An isolated fiber would contribute significantly fewer calories to the cereal, and less change to food properties.

Addition of insoluble fibers to a breakfast cereal will increase the amount of water needed to process it, water that ultimately must be removed, adding process complexity and costs. Viscous soluble fibers added to cereals may increase the viscosity of the dough stage, making it harder to process through the unit operations needed to make the cereal (extrusion, mixing, pumping, drying) [6]. One approach to reducing the viscosity of soluble fibers is to hydrolyze them into shorter polymers, which then avoids these processing issues.

Novel concentrated fibers are being created by reducing their molecular weights through enzymatic hydrolysis, or by chemical modifications of existing fibers, to facilitate their addition to food, as well as to minimize effects on the finished product $[7,8]$. Overweight women who are conscious of their body shape and desire to lose weight are seeking food solutions. To help these consumers, the food industry is customizing foods that are lower in calories and higher in fiber content, many containing novel fibers $[9,10]$. Viscous high molecular weight soluble fibers added to beverages have been shown to increase satiety [11]. Although they have optimized food quality characteristics, it is not certain whether lower molecular weight modified fibers will still have beneficial effects on satiety.

Two randomized, double-blind, crossover design studies were completed to evaluate the effects of an enzyme-hydrolyzed arabinoxylan from wheat (AXOS) versus an intact arabinoxylan from flax (FLAX) added to a ready-to-eat cereal on the postprandial appetitive, hormonal, and/or metabolic responses in overweight women. Subsequent meal energy intake was also assessed. 


\section{Materials and Methods}

\subsection{Subjects}

For both trials, subjects were overweight women, 18-29 years of age, each with a body mass index (BMI) of $25.0-29.9 \mathrm{~kg} / \mathrm{m}^{2}$, inclusive. Exclusion criteria included recent weight loss of $>4.1 \mathrm{~kg}$ within 4 weeks of screening visit; history or presence of cancer, renal, hepatic, endocrine (including diabetes mellitus), pulmonary, biliary, gastrointestinal, pancreatic, or neurologic disorders; recent use of any weight loss drugs; weight-reducing surgery or a diagnosed eating disorder; and pregnancy or planning to become pregnant during the study period. Subjects were regular consumers of breakfast cereal and did not dislike macaroni and cheese.

Subjects were recruited through approved advertisements, flyers, and emails. Before starting the trial, all applicants completed an assessment for inclusion in the study. This included body weight, vital signs, evaluations of inclusion/exclusion criteria, concomitant medication use, a first day of last menses query, as well as medical history, Eating Habits Questionnaire (to exclude unusual eating patterns), in-clinic urine pregnancy test, and Vein Access Scale assessments. Signed written informed consent for participation in the study was obtained from all subjects before protocol-specific procedures were carried out. Subjects were informed of their right to withdraw from the study at any time. These trials were conducted according to Good Clinical Practice Guidelines, the Declaration of Helsinki (2000), and US 21 CFR. The trials were approved by Quorum Review IRB (25787/1 and 26950/1), an appropriately constituted Institutional Review Board, in accordance with the requirements of 21 CFR 56. For trial 1 and 2, 30 and 36 subjects, respectively, were recruited and completed the studies. Five subjects participated in both trials. Women were only tested during the luteal phase of their menstrual cycles (defined as $\geq$ day 15 , where day 1 = first day of menses) in order to improve the sensitivity of satiety testing $[12,13]$. Therefore, before starting each treatment visit, subjects again underwent assessments of body weight, vital signs, evaluation of inclusion/exclusion criteria, concomitant medication use, and a first day of last menses query. For trial 1, treatment visits were a minimum of 7 days apart and for trial 2, visits were a minimum of 4 days apart to enable more treatment visits within the luteal phase of the menstrual cycle and because 4 days was considered to be a sufficient period for washout of any carryover from the previous treatment.

\subsubsection{Diets}

Wheat bran is a major component of some high-fiber breakfast cereals, with arabinoxylans being the predominant fiber in wheat bran [14]. The hydrolyzed fiber included in this study was a wheat bran arabinoxylan extract (AXOS), enzyme hydrolyzed and purified by a proprietary process and obtained from Fugeia N.V. (Belgium). Several treatment steps designed to remove the digestible carbohydrates, cellulose, and proteins from the bran yielded a concentrated wheat arabinoxylan [8] with a degree of polymerization between 3 and 9. The percent fiber in the hydrolyzed AXOS was determined by AOAC method 2009.01 and was $79.6 \%$ [15].

Since an isolated unhydrolyzed wheat bran arabinoxylan was not available in the quantities needed for this work, a flax seed extract composed of arabinoxylans and rhamnogalacturonan was used. The intact fiber (FLAX) was a flax fiber extract obtained from Biogin Biochemicals Co. Ltd. (China). 
The FLAX was extracted from flax seed mucilage using water and ethanol. The percent fiber in the FLAX was determined by AOAC method 2009.01 and was $81.3 \%$ [15].

\subsubsection{RTEC, Flaked}

RTEC was produced by a proprietary process in the Kellogg Company pilot plant. The main unit operations were cooking under pressure, then cooling and drying the dough, forming pellets, drying and tempering, and finally flaking and toasting. Cooking was done in a batch pressure cooker to heat food to a temperature of $123^{\circ} \mathrm{C}$ and $117 \mathrm{kPa}$ pressure to hydrate the grains. The nutrient composition of the cereals is shown in Table 1 and the formulae are referenced [15]. The RTECs were not fortified and any micronutrient losses should be the same in all 4 RTECs.

Table 1. Nutrient composition of RTE cereals.

\begin{tabular}{ccccc}
\hline RTE Cereals & LF & LF-iso & HF-AXOS & HF-FLAX \\
\hline Flaked Cereal (g) & 100 & 70 & 100 & 100 \\
Water (g) & 220 & 260 & 200 & 200 \\
2\% Milk (g) & 180 & 170 & 200 & 200 \\
Mass (g) & 500 & 500 & 500 & 500 \\
Total Carbohydrate (g) & 95 & 69 & 82 & 82 \\
Fiber (g) & 4 & 3 & 19 & 19 \\
Protein (g) & 13 & 11 & 12 & 12 \\
Lipid (g) & 5 & 4 & 5 & 5 \\
Calories (kcal) & 461 & 345 & 345 & 347
\end{tabular}

LF: low-fiber RTEC; HF-AXOS: high-fiber (HF) RTEC with AXOS fiber; HF-FLAX: high-fiber (HF) RTEC with FLAX fiber; LF-iso: isocaloric RTEC to that of the HF breakfasts.

In trial 1 , subjects consumed $100 \mathrm{~g}$ of one of the following RTECs with $2 \%$ milk on three separate days in random order: low fiber (LF, 4 g); high fiber (HF, 15 g) as AXOS; or high fiber (HF, 15 g) as FLAX. In trial 2, subjects randomly consumed the LF, HF-AXOS, or HF-FLAX RTECs as in trial 1, but also consumed a LF RTEC that was isocaloric to the HF meals (LF-iso); thus, the LF-iso was only $70 \mathrm{~g}$. In both trials, the milk was diluted with water to standardize the weight consumed (500 g/breakfast meal).

\subsubsection{Cereal Flake Texture and Sensory Measurements}

RTEC (59 mL) was placed in a Kramer shear press and peak force (g) was assessed using TX.XT Plus Texture Analyzer (Stable Micro Systems, Surrey, UK) with $50 \mathrm{~kg}$ load cell at a test speed of $2 \mathrm{~mm} / \mathrm{s}$. For milk soaked samples, RTE cereal ( $59 \mathrm{~mL}$ ) was placed into $52 \mathrm{~mL}$ of $2 \%$ milk for $7 \mathrm{~min}$. The soaked sample was assessed at a speed of $5 \mathrm{~mm} / \mathrm{s}$ with a square probe. A trained panel of 8 people also evaluated the three RTEC samples for texture attributes in a sequential monadic fashion both at first bite and after chewing ( $<15$ times). The data were collected by panel consensus [16]. 


\subsection{Trial Design}

Trials 1 and 2 were both randomized, double-blind, placebo-controlled, crossover trials. In both trials, subjects fasted 9-13 h before the start of RTEC product consumption ( $t=0 \mathrm{~min}$ ). Subjects recorded all foods and beverages consumed after $1400 \mathrm{~h}$ the day prior to the first treatment visit. These records were collected and reviewed, and subjects were provided a copy of the record and instructed to replicate the same diet from $1400 \mathrm{~h}$ the day prior to each subsequent treatment visit. Following the overnight fast, the subjects randomly consumed one of the breakfasts on each testing day between $0630 \mathrm{~h}$ and $0930 \mathrm{~h}$. Perceived appetite and satiety were assessed before and after breakfast at specific times throughout the $4.5 \mathrm{~h}$ testing day. After the 120 min rating, subjects were allowed $200 \mathrm{~mL}$ of water. The amount of water consumed was recorded, and subjects were asked to consume a similar amount of water at subsequent treatment visits.

In addition to the previous procedures, trial 2 also included blood sample collection. An intravenous catheter was inserted in the forearm for collection of venous blood. To maintain patency of the intravenous catheter, the catheter was flushed with $10 \mathrm{~mL}$ normal saline solution hourly. A baseline blood sample to assess active ghrelin (acylated form; $n$-octanoic acid on serine 3), active glucagon-like peptide-1 (GLP-17-36), total PYY (PYY3-36 + PYY1-36), glucose, and insulin was drawn at $t=-10 \pm 5 \mathrm{~min}$. Subsequent blood samples were collected at $t=15,30,45,60,90,120,180$, and $240 \pm 5 \mathrm{~min}$.

\subsection{Ad Libitum Lunch}

A macaroni and cheese lunch (40 oz.) was provided in coded ceramic pots to subjects at 240 min after the breakfast meal (Stouffer's Family Size 40 oz., Nestlé USA Inc., Solon, OH, USA). Subjects ate directly from the pots and were provided with a standard amount of water during lunch. The quantity of water consumed was recorded. Subjects were allowed $25 \mathrm{~min}$ for lunch and were instructed to eat until comfortably full. Food was weighed prior to and following consumption, and energy intake was assessed based on nutrition facts panel data (Total Fat 17.0 g, Carbohydrates 30.0 g, Dietary Fiber 2.0 g, Sugars 4.0 g, Protein 15.0 g, 330 kcal/225 g).

\subsection{Appetite Measurements}

Perceived appetite was assessed using a visual analogue scale (VAS) [17]. The scale was $100 \mathrm{~mm}$ in length with descriptions expressing the most positive and most negative ratings for desire to eat, hunger, fullness, and prospective consumption. Subjects placed a mark crossing the line connecting descriptors and the distance in $\mathrm{mm}$ from the left side was used as the rating. Perceived appetite was assessed prior to breakfast meal consumption ( $t=-15$ and $-5 \mathrm{~min}$ ). At $t=0 \mathrm{~min}$, subjects consumed one of the RTECs between 0700 and $1000 \mathrm{~h}$. Subjects were allowed $15 \mathrm{~min}$ to consume the breakfast meal. Subsequent VAS ratings were completed by the subjects at $t=15,30,45,60,75,90,105,120,135,150,165,180,195$, 210, 225, 240, and $270 \mathrm{~min}$. Net incremental area under the curve (niAUC) was calculated using the trapezoid method for $2 \mathrm{~h}$ and $4 \mathrm{~h}$. 


\subsection{Hormonal Analyses}

Blood was collected in test tubes containing EDTA and protease inhibitors (AEBSF and DPP-IV) to reduce protein degradation. Within $10 \mathrm{~min}$ of collection, samples were centrifuged at $4{ }^{\circ} \mathrm{C}$ for $10 \mathrm{~min}$. The plasma was separated and stored at $-80{ }^{\circ} \mathrm{C}$ for future analysis. Plasma active ghrelin (acylated form; n-octanoic acid on serine 3), active GLP-1 (GLP-17-36), total PYY (PYY3-36 + PYY 1-36), and insulin, were prepared using the Milliplex MAP magnetic bead-based multi-analyte, metabolic panel, 4-plex immunoassay (HMHMAG-34K; Millipore, St. Charles, MO, USA) and measured using Luminex Magpix with xPONENT software (Luminex Corporation, Austin, TX, USA). Intra- and inter-assay CV were 2\% and $8 \%, 7 \%$ and $10 \%, 2 \%$ and $11 \%$, and $3 \%$ and $6 \%$ for active ghrelin (acylated form), active GLP-1 (GLP-17-36), total PYY (PYY3-36 + PYY1-36), and insulin, respectively. The detection limit of the assay was 2, 7, 8, and $58 \mathrm{pg} / \mathrm{mL}$ for active ghrelin (acylated form), active GLP-1 (GLP-17-36), total PYY (PYY3-36 + PYY1-36), and insulin, respectively. Plasma glucose was measured with a glucose oxidase assay using a BioTEk ELx808 Absorbance Microplate Reader (BioTek, Winooski, VT, USA) and Glucose Oxidase Reagent (TR-15221; ThermoFisher Scientific, Austin, TX, USA). Intra- and inter-assay CV for glucose were $2 \%$ and $5 \%$. The detection limit of the assay was $1.0 \mathrm{mg} / \mathrm{dL}$.

\subsection{Gastrointestinal Tolerability}

Assessment was by self-reported scoring on a 6-point scale with 0 being not experienced, and 5 being most severe experience for each attribute [18]. Five different gastrointestinal symptoms were measured (cramping, flatulence, gas/bloating, loose stools, and nausea).

\subsection{Statistics}

\subsubsection{VAS Appetite Scores}

All values are reported as means with their respective standard errors. Statistical analyses were conducted using SAS for Windows (version 9.1.3 or higher, Cary, NC, USA). Descriptive statistics are presented for the outcome parameters for each RTE cereal. Response differences among RTE cereals were assessed using repeated measures analysis of variance (ANOVA) including subject as a random variable, and test diet as a fixed effect. The model was reduced until only test diet and any significant $(p<0.05)$ terms remained. Pairwise comparisons between all treatment conditions were conducted using Tukey's adjustment for multiple comparisons.

All tests of significance, unless otherwise stated, were performed at $\alpha=0.05$, two-sided. Assumption of normality of residuals from the final model of each outcome parameter was investigated by the Shapiro-Wilk test [19].

\subsubsection{Hormones and Glucose}

All values are reported as means with their respective standard errors. Statistical analyses were conducted using PROC MIXED in SAS for Windows (version 9.2, Cary, NC, USA). Repeated measures over time were modeled with a heterogeneous banded (Toeplitz) covariance structure using the repeated statement of SAS. For all effects, significance was declared at $p<0.05$. Descriptive statistics are presented 
for the outcome parameters for each test diet using least squares means. Response differences among breakfast meals were assessed for each time point using mixed model analysis including subject as a random variable, and treatment as a fixed effect. The model was reduced until only test diet and any significant $(p<0.05)$ terms remain. Pairwise comparisons between all treatment conditions were conducted using Tukey's adjustment for multiple comparisons. Net incremental area under the curve (niAUC) was calculated using the trapezoid method and results reported at 2 and $4 \mathrm{~h}$ [20]. Per protocol, hormone analysis values were screened to reject subjects with at least 1 plasma hormone niAUC value greater than 3 times the standard deviation from the mean in at least 2 breakfast meals. One subject did not complete one of the study treatment testing days and thus had no hormonal or glucose data, and one subject was excluded from the glucose data analyses since her fasting plasma glucose concentration was greater than $100 \mathrm{mg} / \mathrm{dL}$. Lastly, three subjects displayed GLP-1 concentrations that were below the assay limit of detection. Thus, the number of data points per hormone and per breakfast meal varied.

\subsubsection{Sample Size}

In trial 1, with an evaluable sample of 30 subjects, the study was projected to have $80 \%$ power to detect a difference of $10 \mathrm{~mm}$ in the appetite score (pre-meal to $240 \mathrm{~min}$ ) between treatments assuming a standard deviation of $15.9 \mathrm{~mm}$ based on previous work completed by the investigators. This assumes a nominal $p$-value of 0.017 to account for up to three primary comparisons using a Šidák adjustment [21]. Rolling recruitment was employed until the desired 30 subjects had been randomized. In trial 2, with an evaluable sample of 33 subjects, the study was projected to have $80 \%$ power to detect a difference of $9.8 \mathrm{~mm}$ in the average appetite composite score (pre-meal to $240 \mathrm{~min}$ ) between treatments assuming a standard deviation of $15.9 \mathrm{~mm}$. This assumes a nominal p-value of 0.0127 to account for up to four primary comparisons using a Šidák adjustment. A sample of 36 subjects was randomized to allow for attrition and non-compliance. After consuming control A RTE cereal, 27 subjects were included for GLP-1, and 30 subjects for the remaining hormone assays and glucose. After consuming the remaining breakfast RTE cereals, 28 subjects were included for GLP-1, and 31 subjects for the remaining hormone assays and glucose.

\section{Results}

\subsection{Cereal Flake Texture and Sensory Characteristics}

The composition of RTECs was similar in protein, fat (Table 1), and flake density (133, 136, and $166 \mathrm{~kg} / \mathrm{m}^{3}$ for the LF, HF-AXOS, and HF-FLAX RTECs, respectively) to minimize the impact of these factors in the comparisons. RTEC texture was evaluated by both instrumental and sensory methods. The 3 RTECs had similar texture based on peak force measurements both dry and in milk (dry: $9744 \pm 1158$, $7882 \pm 722$, and $7369 \pm 1288 \mathrm{~g}$ of force, and in milk: 35,588 \pm 7305, 35,506 \pm 8196, and 30,667 $\pm 3598 \mathrm{~g}$ of force for LF, HF-AXOS and HF-FLAX RTECs, respectively). There were slight texture differences in sensory evaluation of the 3 RTE cereals (Table 2). The LF flake exhibited a more cohesive mass after chewing and in milk. The HF-AXOS RTEC absorbed the least moisture/saliva after chewing. Finally, the HF-FLAX RTEC was the least gritty. Flavor was also judged to be very similar (data not shown). 
Table 2. Sensory characteristics of RTE flaked cereal dry and with milk. Results were collected as panel consensus ${ }^{\text {a }}$.

\begin{tabular}{llll}
\hline Attribute Title & LF & HF-AXOS & HF-FLAX \\
\hline First Chew (Dry Cereal) & & & \\
Hardness, Molars & 5.0 & 5.5 & 5.0 \\
Fracturability & 4.5 & 5.0 & 4.0 \\
\hline Chew Down (Dry Cereal) & & & \\
Moisture Absorption & 11.0 & 7.5 & 10.0 \\
Cohesiveness of Mass & 8.5 & 7.5 & 8.0 \\
Gritty/Particles & 4.0 & 3.0 & 2.0 \\
\hline Min in Milk & & & \\
Fracturability & 0.0 & 0.0 & 0.0 \\
Cohesiveness of Mass & 5.5 & 4.5 & 4.0 \\
\hline
\end{tabular}

a 15-point scale divided into $1 / 2$ point increments, with 0 meaning "none" and 15 meaning "extremely strong” [16] LF: low-fiber RTEC; HF-AXOS: high-fiber (HF) RTEC with AXOS fiber; HF-FLAX: high-fiber (HF) RTEC with FLAX fiber.

\subsection{Subjects}

Subject characteristics for each trial are outlined in Table 3. Characteristics of the participants were controlled with inclusion of females within a narrow range of BMI and age. Since the high fiber diets contained $19 \mathrm{~g}$ of fiber per serving (15 $\mathrm{g}$ test fiber and $4 \mathrm{~g}$ base cereal fiber), gastrointestinal tolerability was assessed in both trials (data not presented for trial 1) at each treatment [18].

Table 3. Subject characteristics at baseline.

\begin{tabular}{ccc}
\hline Parameter & Trial 1 & Trial 2 \\
\hline Female & 30 & 36 \\
Race/ethnicity & & \\
Non-Hispanic White & 20 & 23 \\
Black/African American & 8 & 8 \\
Asian or Pacific Islander & 1 & 2 \\
Multiracial & 1 & 3 \\
Smoking Status & & \\
Non-Smoker & 24 & 27 \\
Current Smoker & 2 & 5 \\
Past Smoker & 4 & 4 \\
Age (years) & $22.5(0.6)^{\text {a }}$ & $24.3(0.5)$ \\
Weight (kg) & $72.9(1.2)$ & $74.3(1.2)$ \\
Body Mass Index (kg/m $\left.{ }^{2}\right)$ & $27.0(0.3)$ & $27.4(0.3)$ \\
Systolic Blood Pressure (mm Hg) & $109(2)$ & $113(2)$ \\
Diastolic Blood Pressure (mm Hg) & $66(1)$ & $71(2)$ \\
Heart Rate (bpm) & $73(2)$ & $77(2)$ \\
\hline
\end{tabular}

${ }^{\mathrm{a}}$ standard error of mean in parentheses. 
Gastrointestinal symptoms were not observed in a majority (66\%-75\%) of the participants. However, for those who did experience symptoms during the study, flatulence was higher with the high-fiber containing RTECs ( $1.3 \pm 0.3$ and $1.1 \pm 0.3$ for HF-AXOS and HF-FLAX RTECs, respectively) compared with both control diets ( $0.3 \pm 0.2$ and $0.2 \pm 0.1$ for LF and LF-iso RTECs, respectively). Gas/bloating experienced was also higher with the high-fiber containing RTECs $(1.5 \pm 0.3$ and $1.4 \pm 0.3$ for HF-AXOS and HF-FLAX RTECs, respectively) compared with both LF controls $(0.3 \pm 0.2$ and $0.1 \pm 0.1$ for LF and LF-iso RTECs, respectively).

\subsection{Trial 1}

Increasing fiber content from $4 \mathrm{~g}$ /serving in the control cereal to $19 \mathrm{~g} /$ serving in the two high-fiber cereals did not affect perceived appetite ratings. Results for VAS scores (mm) of Hunger, Fullness, Desire to Eat, and Prospective Consumption showed no significant differences $(p>0.05)$ among treatment conditions in appetite ratings at any time point (only Hunger data presented, Figure 1). Total niAUC ${ }_{0-240}$ values for Hunger over the pre ad libitum lunch phase were $-8379 \pm 1159,-7064 \pm 1211$, and $-7928 \pm 1234 \mathrm{~mm} \times$ minutes \pm SEM for the control, AXOS, and FLAX RTE cereals, respectively. No significant differences were observed among conditions.

The energy intake subjects consumed during the ad libitum lunch did not differ significantly among treatments $(p=0.96)$, and thus subjects did not compensate for lower calorie intakes with the high-fiber breakfasts (breakfast calories 461, 345, 347 kcal for LF, HF-AXOS and HF-FLAX RTECs, respectively). Mean energy intakes during the ad libitum lunch were $552 \pm 37,545 \pm 37$, and $559 \pm 36 \mathrm{kcal} \pm \mathrm{SEM}$ for the LF, HF-AXOS, and HF-FLAX RTE cereals, respectively. Mean total energy intakes from the combined RTEC breakfast and lunch meals were $1013 \pm 36,907 \pm 37$, and $906 \pm 36 \mathrm{kcal} \pm$ SEM for the LF, HF-AXOS, and HF-FLAX RTECs, respectively ( $p=0.065$ across breakfasts). Consequently participants consumed $\sim 10 \%$ fewer kcal for breakfast and lunch combined when fed the HF-AXOS ( $p=0.109)$ and HF-FLAX ( $p=0.101)$ RTECs versus the LF RTEC because the LF RTEC breakfast contained more calories. When both fiber treatments were averaged together, the difference in energy intake reached significance versus control $(p=0.036)$.

\subsection{Trial 2}

In this trial, 3 of the diets were the same as in trial 1. An additional control was added to compare a low-fiber control RTEC with high calories (LF; $4 \mathrm{~g}$ fiber; $461 \mathrm{kcal}$ ), to a low-fiber control RTEC (LF-iso; $3 \mathrm{~g}$ fiber; $341 \mathrm{kcal}$ ) energy matched to the high-fiber RTECs. As with trial 1, increasing fiber content from 3 to $4 \mathrm{~g}$ /serving in the two control cereals to $19 \mathrm{~g} /$ serving in the two high-fiber cereals did not affect appetite ratings. Results for VAS scores of Hunger, Fullness, Desire to Eat, and Prospective Consumption showed no significant differences $(p>0.05)$ among treatment conditions in appetite ratings at any time point (only Hunger data presented, Figure 1). Total niAUC0-240 values for Hunger over the pre ad libitum lunch phase were $-10,342 \pm 1054,-9566 \pm 1037,-10,723 \pm 1060,-9691 \pm 1046 \mathrm{~mm}$ $\times$ minutes \pm SEM for the LF, LF-iso, HF-AXOS, and HF-FLAX RTECs, respectively. No significant differences were observed among RTECs. 

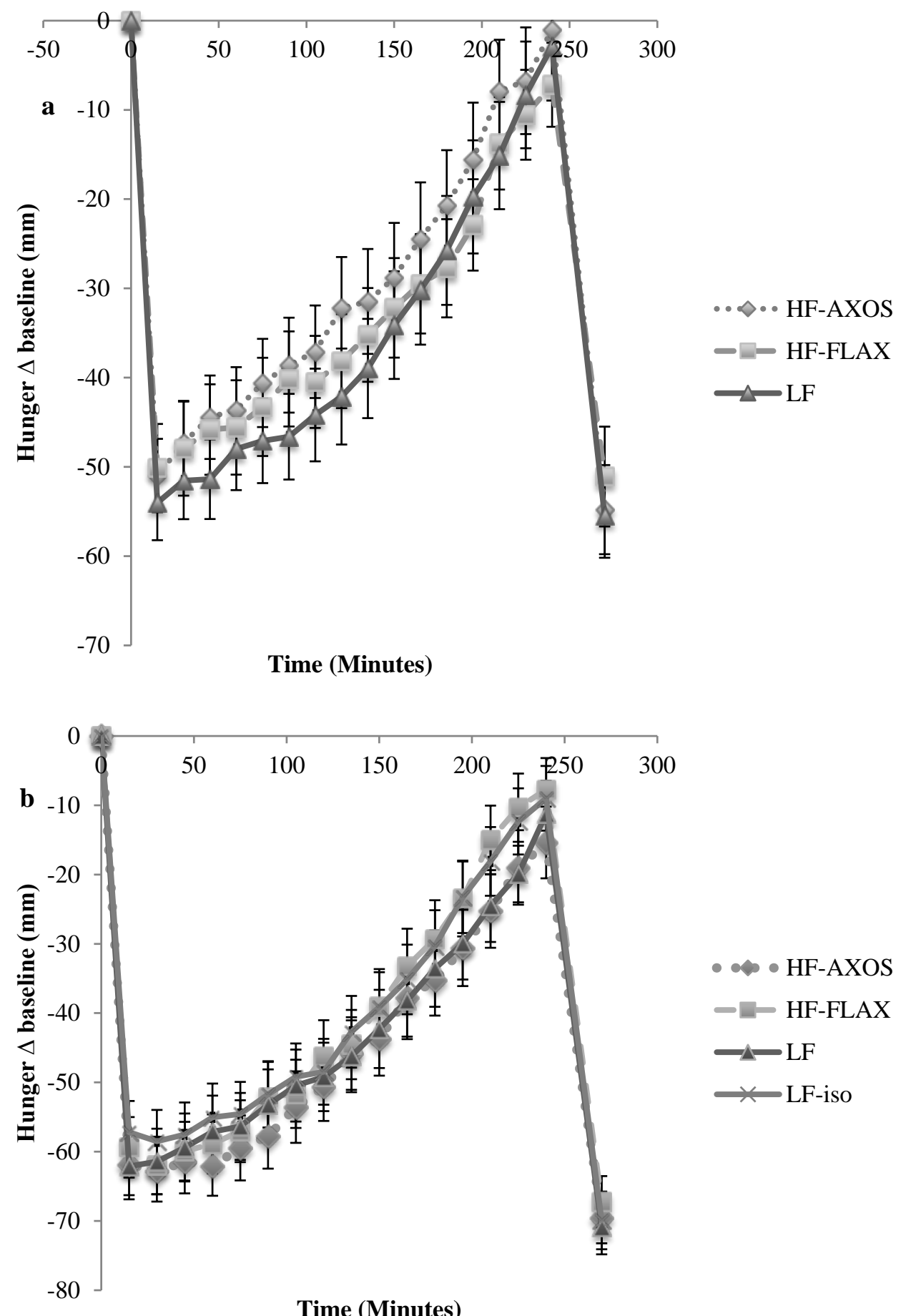

Figure 1. Hunger Score change from baseline following consumption of each breakfast. (a) Clinical Trial 1. Data are presented as mean \pm SEM, based on 30 subjects. (b) Clinical Trial 2. Data are presented as mean \pm SEM, based on 36 subjects. There were no significant differences between treatments at any time point $(p>0.05)$ in either trial. LF: low-fiber RTEC; HF-AXOS: high-fiber (HF) RTEC with AXOS fiber; HF-FLAX: high-fiber (HF) RTEC with FLAX fiber; LF-iso: isocaloric RTEC to that of the HF breakfasts. 
As in trial 1, subjects consumed similar amounts of macaroni and cheese during the ad libitum lunch in the four treatment conditions. Again, just as in the first clinical trial, they did not compensate for the lower calories in the isocaloric breakfasts (461, 341, 345, $347 \mathrm{kcal}$ for the LF, LF-iso, HF-AXOS and HF-FLAX RTECs, respectively). Energy intakes from lunch were $533 \pm 37,558 \pm 38,544 \pm$ 37, and $547 \pm 36 \mathrm{kcal}$ (mean \pm SEM) for the LF, LF-iso, HF-AXOS, and HF-FLAX RTECs, respectively and were not significantly different between treatments $(p=0.77)$. Total energy intakes from the combined RTEC and lunch for the LF-iso $899 \pm$ 38, HF-AXOS $907 \pm$ 37, and HF-FLAX $894 \pm 36$ kcal (mean \pm SEM) conditions were significantly lower than for LF, $994 \pm 37 \mathrm{kcal}$ ( $p \leq 0.006$ for each), again suggesting that the participants did not compensate for the lower energy intake at the breakfast meal.

Since the 2 high-fiber cereals and the high-energy control cereal were the same in both clinical trials, the niAUC data were pooled for these test conditions. Although this served to increase the number of subjects included in the analysis $(n=65)$, there was still no significant difference in appetite among these test cereals $(p>0.05)$ at any time point.

\subsection{Hormones and Glucose}

Blood samples were collected from each of the subjects after the overnight fast prior to each day they ate the 4 different breakfasts. Five subjects were removed from the data pool per protocol. One subject was removed due to high fasting plasma glucose concentrations ( $>200 \mathrm{mg} / \mathrm{dL}$ ) on all four days. The other 4 subjects were removed because one or more of their niAUC hormone concentrations were $>3$ standard deviations from the mean for at least 2 breakfast meals. Baseline values for each of the hormones and glucose, after these subjects were removed, were similar prior to consumption of the 4 breakfasts. Fasted average glucose concentrations were in the normal range, consistent with screening criteria for the study to exclude subjects that had diabetes or pre-diabetes.

\subsubsection{Ghrelin}

Similar post-meal reductions in plasma ghrelin were observed for all 4 breakfast meals (Figure 2a). Ghrelin concentrations all decreased during the first $45 \mathrm{~min}$. Ghrelin then began to return to baseline values at approximately $60 \mathrm{~min}$ and continued to increase until lunch was consumed. The greatest reduction of ghrelin after consumption of breakfast, as well as the longest delay to return to baseline concentrations, tended to occur when subjects consumed the high-energy LF RTEC. A treatment $\times$ time interaction was observed with repeated measures, $p=0.01$. At $t=45$ and $t=90$ min, ghrelin concentrations in subjects consuming LF RTEC were significantly lower $(p<0.05)$ than when they consumed HF-FLAX RTEC. At $t=120 \mathrm{~min}$, ghrelin concentration measurements for subjects consuming LF RTEC were significantly lower $(p<0.05)$ than when they consumed LF-iso and HF-AXOS RTECs. At $t=180$ min, ghrelin concentrations in subjects consuming LF RTEC were significantly lower $(p<0.05)$ than when they consumed LF-iso RTEC. Composition of the RTECs did not affect mean niAUC measurements of plasma ghrelin concentrations in subjects after breakfast consumption (Table 4). 

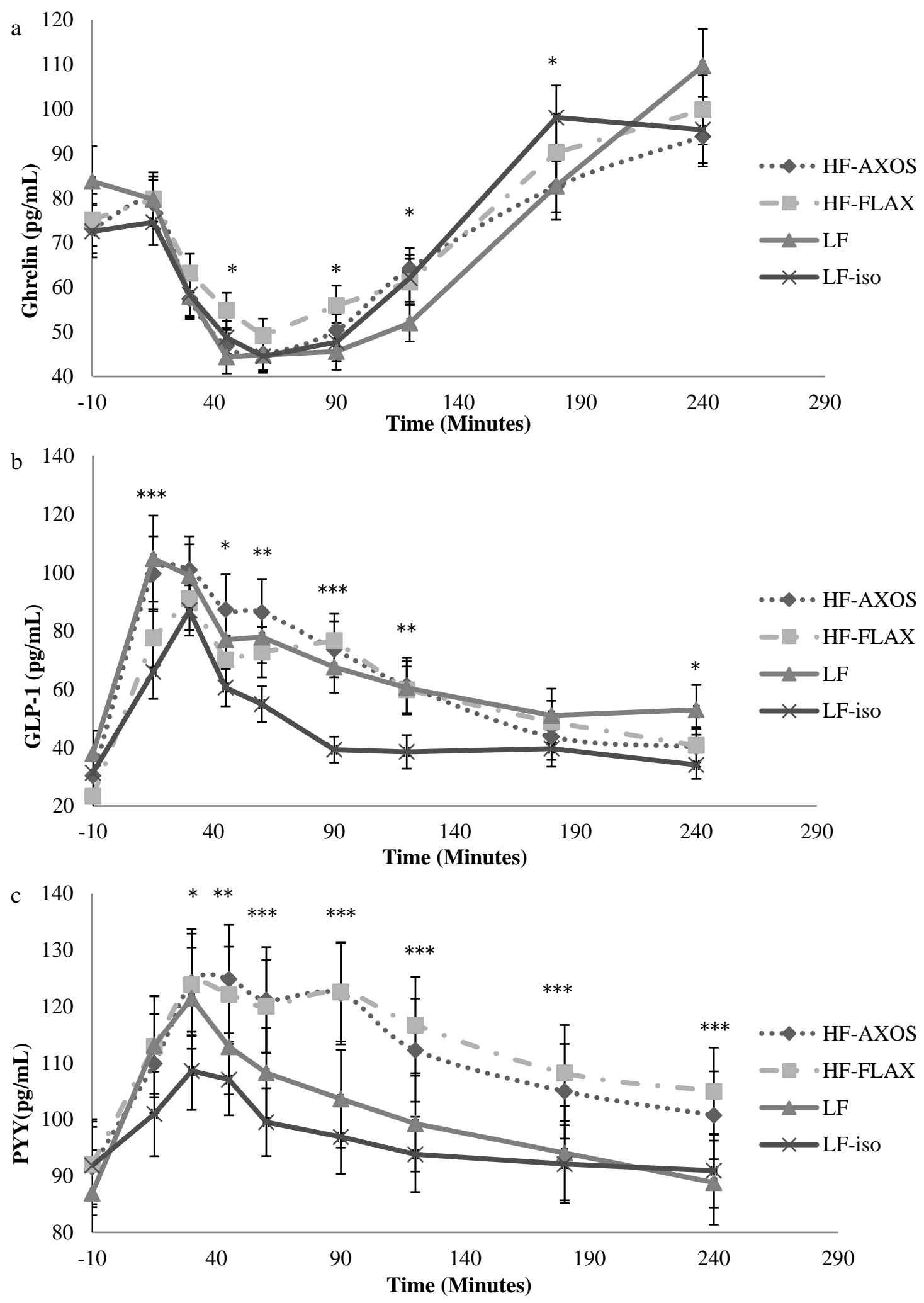

Figure 2. Plasma hormone concentrations at selected time points: (a) active ghrelin, (b) active GLP-1, (c) total PYY. Significant differences among groups are signified as follows: $* * * p<0.001$; ${ }^{* *} p<0.01$; $* p<0.05$. LF: low-fiber RTEC; HF-AXOS: high-fiber (HF) RTEC with AXOS fiber; HF-FLAX: high-fiber (HF) RTEC with FLAX fiber; LF-iso: isocaloric RTEC to that of the HF breakfasts. 
Table 4. Net incremental area under the curve (niAUC) for each hormone at $2 \mathrm{~h}$ and at $4 \mathrm{~h}$ : (a) 2 h niAUC, (b) 4 h niAUC.

\begin{tabular}{|c|c|c|c|c|c|}
\hline $\begin{array}{c}\text { Blood Parameter }{ }^{a} \\
\left(\text { mean } \times 10^{3}\right)\end{array}$ & $\mathbf{L F}$ & LF-iso & HF-AXOS & HF-FLAX & $p$-Value \\
\hline \multicolumn{6}{|l|}{ (a) } \\
\hline Ghrelin $(\mathrm{pg} / \mathrm{mL} \times \min )^{b}$ & $6.45(.51)^{\mathrm{A}}$ & $6.77(.51)^{\mathrm{A}}$ & $6.67(.51)^{\mathrm{A}}$ & $7.19(.51)^{\mathrm{A}}$ & 0.33 \\
\hline GLP-1 $(\mathrm{pg} / \mathrm{mL} \times \min )$ & $8.81(1.11)^{\mathrm{A}}$ & $6.27(1.11)^{\text {В }}$ & $9.25(1.11)^{\mathrm{A}}$ & $8.35(1.11)^{\mathrm{A}}$ & $<0.01$ \\
\hline Total PYY $(\mathrm{pg} / \mathrm{mL} \times \min )$ & $12.76(1.02){ }^{\mathrm{AB}}$ & $11.87(1.01)^{\mathrm{B}}$ & $13.93(1.02)^{\mathrm{A}}$ & $13.93(1.01)^{\mathrm{A}}$ & $<0.01$ \\
\hline Glucose $(\mathrm{mg} / \mathrm{dL} \times \min )$ & $11.59(.32)^{\mathrm{A}}$ & $11.80(.32)^{\mathrm{A}}$ & $11.29(.32)^{\mathrm{A}}$ & $11.18(.32)^{\mathrm{A}}$ & 0.052 \\
\hline Insulin $(\mathrm{pg} / \mathrm{mL} \times \min )$ & $327.90(32.62)^{\mathrm{A}}$ & $299.80(32.52){ }^{\mathrm{AB}}$ & $282.80(32.62)^{\mathrm{B}}$ & $269.00(32.52)^{\text {в }}$ & $<0.01$ \\
\hline \multicolumn{6}{|l|}{ (b) } \\
\hline Ghrelin $(\mathrm{pg} / \mathrm{mL} \times \min )$ & $11.32(.88)^{\mathrm{A}}$ & $12.06(.88)^{\mathrm{A}}$ & $11.40(.89)^{\mathrm{A}}$ & $12.25(.88)^{\mathrm{A}}$ & 0.33 \\
\hline GLP-1 $(\mathrm{pg} / \mathrm{mL} \times \min )$ & $12.06(1.56)^{\mathrm{A}}$ & $8.27(1.56)^{\mathrm{B}}$ & $11.96(1.56)^{\mathrm{A}}$ & $11.27(1.55)^{\mathrm{A}}$ & $<0.01$ \\
\hline Total PYY $(\mathrm{pg} / \mathrm{mL} \times \min )$ & $18.41(1.49)^{\mathrm{BC}}$ & $17.40(1.49)^{\mathrm{C}}$ & $20.19(1.49)$ АВ & $20.44(1.49)^{\mathrm{A}}$ & $<0.01$ \\
\hline Glucose $(\mathrm{mg} / \mathrm{dL} \times \min )$ & $16.16(.37)^{\mathrm{A}}$ & $16.41(.36)^{\mathrm{A}}$ & $16.01(.37)^{\mathrm{A}}$ & $15.75(.36)^{\mathrm{A}}$ & 0.12 \\
\hline Insulin $(\mathrm{pg} / \mathrm{mL} \times \min )$ & $436.00(42.19)^{\mathrm{A}}$ & $382.30(42.08)^{\text {в }}$ & $360.30(42.19)^{\text {B }}$ & $348.70(42.08)^{\text {В }}$ & $<0.01$ \\
\hline \multicolumn{6}{|c|}{${ }^{a}$ number of subjects varied: LF—GLP-1, 27; Ghrelin, Total PYY, Glucose, Insulin, 30; remaining breakfasts—GLP-1, 28; Ghrelin, Total } \\
\hline
\end{tabular}

\subsubsection{GLP-1}

GLP-1 concentrations immediately increased after consumption of each of the 4 breakfast meals, peaked after $30 \mathrm{~min}$, and slowly decreased towards baseline, remaining above baseline values throughout the remainder of the testing day (Figure 2b). Overall, GLP-1 concentrations tended to be lower when LF-iso RTEC was consumed. When subjects consumed LF-iso RTEC, plasma concentrations of GLP-1 were significantly lower $(p<0.05)$ than when subjects consumed HF-FLAX RTEC at $t=90$ and 120 min. Concentrations of GLP-1 were lower in subjects consuming LF-iso RTEC than when they consumed LF RTEC at $t=15,90,120$, and $240 \mathrm{~min}$. Concentrations of GLP-1 were also lower in subjects when they consumed LF-iso RTEC than when they consumed HF-AXOS RTEC, with significant differences $(p<0.05)$ in the later portion of the time curve at $t=15,45,60,90$, and $120 \mathrm{~min}$. Concentrations of GLP-1 were the same in subjects consuming LF RTEC as when subjects consumed either the HF-AXOS or the HF-FLAX RTEC at all time points. Mean niAUC measurements of GLP-1 values demonstrated that subjects consuming LF-iso RTEC had a lower plasma concentration of GLP-1 than when consuming the other 3 treatment cereals at 2 and $4 \mathrm{~h}$ (Table 4).

\subsubsection{PYY}

PYY concentrations immediately increased after consumption of each of the 4 breakfast meals, peaked after $30 \mathrm{~min}$, and then slowly decreased throughout the remainder of the testing day (Figure 2c). A treatment-time interaction was observed with repeated measures, $p=0.01$. Subjects consuming the two high-fiber RTECs had significantly higher $(p<0.05)$ PYY concentrations than when they consumed 
the two LF cereals, suggesting a fiber effect on PYY concentrations. Subjects consuming HF-AXOS RTEC also had plasma PYY concentrations higher than when they consumed LF-iso RTEC at almost every time point, with significant differences $(p<0.05)$ at $t=30,45,60,90,120$, and $180 \mathrm{~min}$. Overall, PYY concentrations tended to be lowest when subjects consumed LF-iso RTEC. When subjects consumed HF-FLAX RTEC, PYY concentrations were higher than when they consumed LF-iso, with significant differences $(p<0.05)$ at $t=30,45,60,90,120,180$, and $240 \mathrm{~min}$. When subjects consumed LF-iso RTEC, they tended to have lower plasma PYY concentrations than when they consumed the high-energy LF RTEC. Subjects consuming HF-AXOS RTEC had a significantly higher $(p<0.05)$ PYY concentration than when they consumed LF RTEC at $t=90$. niAUC measurements of PYY concentrations were higher in subjects consuming HF-FLAX RTEC than when they consumed LF-iso RTEC at $2 \mathrm{~h}$, and when they consumed LF-iso or LF RTECs at $4 \mathrm{~h}$ (Table 4). Mean niAUC measurements of PYY concentrations were also higher in subjects consuming HF-AXOS RTEC than when they consumed LF-iso RTEC at both 2 and $4 \mathrm{~h}$.

\subsubsection{Glucose and Insulin}

Glucose concentrations increased immediately after consumption of each of the 4 breakfast meals, reached a peak at $30 \mathrm{~min}$, and then decreased during the remainder of the test (Figure 3a). Subjects' glucose concentrations all returned to baseline between 120 and $180 \mathrm{~min}$. Plasma glucose concentrations were higher when subjects consumed LF-iso than when they consumed HF-FLAX RTEC, with significant differences $(p<0.05)$ at $t=45$ and $60 \mathrm{~min}$. Glucose concentrations in subjects consuming all 4 breakfast cereals were not significantly different at the other time points. Mean niAUC measurements of glucose concentrations of subjects consuming all 4 RTECs were not statistically different at 2 or $4 \mathrm{~h}$ (Table 4).

Insulin concentrations also increased immediately after consumption of each of the 4 breakfast meals, reaching a peak between 30 and $45 \mathrm{~min}$, and then decreased during the remainder of the test (Figure 3b). Unlike glucose, subjects' insulin concentrations did not return to baseline until approximately $240 \mathrm{~min}$. Insulin concentrations were higher in subjects consuming the high energy LF RTEC than when they consumed the other breakfasts, with significant differences $(p<0.05)$ versus LF-iso RTEC at $t=120$ and 180 min; versus HF-AXOS RTEC at $t=90,120,180$, and $240 \mathrm{~min}$; and versus HF-FLAX RTEC at time points $t=60,90,120$, and $180 \mathrm{~min}$. Mean niAUC measurements of insulin concentrations were higher in LF RTEC than HF-AXOS and HF-FLAX RTECs at $2 \mathrm{~h}$ and higher than LF-iso, HF-AXOS, and HF-FLAX RTECs at $4 \mathrm{~h}$ (Table 4). 

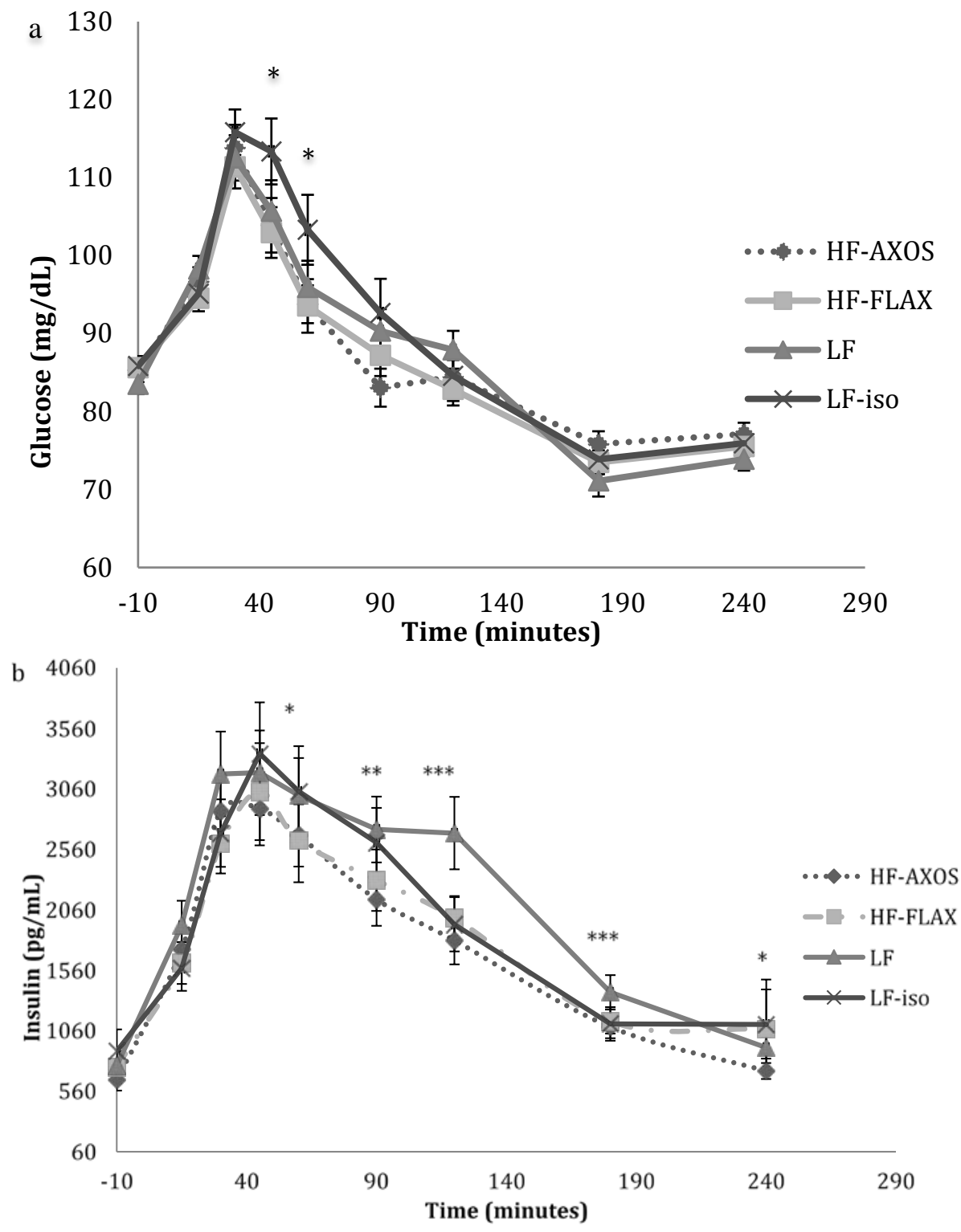

Figure 3. Plasma glucose and insulin concentrations at selected time points: (a) glucose, (b) insulin. Significant differences among groups are signified as follows: *** $p<0.001$; $* * p<0.01$; $* p<0.05$. LF: low-fiber RTEC; HF-AXOS: high-fiber (HF) RTEC with AXOS; HF-FLAX: high-fiber (HF) RTEC with FLAX fiber; LF-iso: isocaloric RTEC to that of the HF breakfasts.

\section{Discussion}

The effects of increased dietary fiber on appetite control and satiety are inconsistent, potentially due to type of fiber, dosage, and delivery form incorporated in these studies as well as the physicochemical properties of the fibers [1-3]. Also, fibers can be modified by hydrolysis to facilitate incorporation into food, making their effects on satiety even more uncertain. It is important to further understand how the physicochemical properties of fiber and fiber modifications influence satiety and energy intake parameters. This study was designed to evaluate the effects of an enzyme-hydrolyzed arabinoxylan from wheat (AXOS) versus an intact arabinoxylan from flax (FLAX) added to a RTEC on perceived appetite, on subsequent meal energy intake, and on hormonal responses in overweight women. No differences in 
perceived appetite and satiety or subsequent meal energy intake were observed between the low-fiber versus the high-fiber breakfast meals in both trials. However, the high fiber breakfast meals led to elevated plasma GLP-1 and PYY concentrations compared to the low-fiber versions. This was an acute study design; longer-term studies are needed to further understand these physicochemical properties of fiber on satiety.

\subsection{Perceived Appetite}

There have been numerous studies comparing perceived appetite after consuming RTECs with added fiber. No effect was found in perceived hunger or fullness (AUC) when comparing RTEC with $41 \mathrm{~g}$ fiber, $250 \mathrm{kcal}$, and a $550 \mathrm{~g}$ meal size with a low-fiber control cereal (1.5 g fiber) 60 or 120 min after the breakfast meal [22]. Samra and Anderson [23] provided a RTEC with $33 \mathrm{~g}$ fiber and a meal size of $500 \mathrm{~g}$, and found that average appetite scores 75 min later were not statistically different versus a low-fiber (1.5 g) RTE cereal. They did observe an increase in fullness with the higher fiber RTEC. Other studies compared RTECs with lower fiber concentrations on perceived appetite [24-27]. The majority of these studies indicate that there was no effect of fiber on perceived appetite in this food form. The results from the current study support this conclusion. This is in conflict with many studies where a modest amount of fiber added to beverages increased perceived appetite [11]. Food form may have an impact on the ability of fiber to increase satiety.

In a beverage system, as little as $2.5 \mathrm{~g}$ fiber has been shown to affect perceived appetite [28,29]. Significant hunger reduction $(-13 \%)$ with a psyllium $(7.4 \mathrm{~g})$ containing beverage relative to no fiber control was found [30]. These studies demonstrated a perceived appetite effect of beverages at much lower added fiber levels than we used in RTECs. One possible explanation is that fibers need to be hydrated before their viscous properties can be fully realized, and this is more likely to occur in a beverage food form than a solid one, or than in a meal with liquid added to a solid form, like milk added to RTEC. In the current study $\sim 80 \%$ of the meal mass was liquid (Table 1 ); however, the physical form of the RTE flake may have attenuated the fiber's ability to affect perceived appetite.

Viscosity of the fiber is believed to be responsible for some of the satiety effects of beverages [11,29,31]. Kristensen and Jensen [32] reviewed nine published studies on the effect of fiber and viscosity on satiety of beverages. They found that in most cases increased viscosity led to increased satiety with fiber levels tested ranging from 3 to $10 \mathrm{~g}$. Many of these beverage studies used very viscous fibers like alginates and gums that have a very high molecular weight and impact viscosity at a low concentration. The FLAX ingredient we used to produce the high-fiber RTEC had a high molecular weight and a very high viscosity [15]. We anticipated that it would produce a higher viscosity RTEC; however, we found that processing reduced the FLAX fiber molecular weight and viscosity of the cereal. The most viscous cereal at the time of consumption was the LF RTEC (from the higher available gelatinized starch present); however, that viscosity might quickly be reduced by digestive amylases in the intestine. Most RTEC studies used a low-fiber, corn flake control RTEC that had a relatively high level of gelatinized starch. Although viscosity of cereal is seldom reported, high-fiber RTECs may not be more viscous than low-fiber, high-starch control RTECs. This may contribute to the reported inconsistency of high-fiber cereals to enhance satiety. 


\subsection{Lunch Meal Energy Intake}

The overweight female subjects in the current study were offered the ad libitum lunch meal $4 \mathrm{~h}$ after the breakfast meals because this represented a typical time interval between breakfast and lunch meals for most adults. In the current study, subjects consumed a similar amount of calories at lunch regardless of breakfast calories consumed. By $4 \mathrm{~h}$, the effects of the breakfast cereals at the lunch meal may have been diminished, since perceived appetite scores were almost at baseline. In addition, both clinical trials studied overweight female subjects, who may be less responsive to appetite cues [33]. Other investigators also found similar energy intake at lunch offered between 3 and $3.5 \mathrm{~h}$ after subjects consumed RTEC with high fiber content [24,25]. However, unlike these studies, some investigators using RTEC did observe a reduction in second meal energy intake when they employed a shorter time interval between breakfast and lunch $[23,27,34]$. This may explain why they observed a lower energy intake in the second meal.

While we cannot rule out the possibility that the high palatability of the macaroni and cheese lunch meal could also have been a factor biasing toward high energy intake and failure to show a difference between conditions in energy intake despite differences in appetite-related hormones (discussed below), it seems unlikely to have been a material issue influencing the results, given that both VAS and energy intake results were concordant in both trials. It is also possible that the subjects stopped eating due to sensory-specific satiety given they were able to consume only one type of food, contributing to the discrepancy between the hormone and the food intake data [35].

\subsection{GLP-1 and PYY}

Plasma GLP-1 and PYY concentrations were significantly higher with the high-fiber breakfasts than with an equal calorie low-fiber control RTEC. Secretion of GLP-1 and PYY has been shown to be co-located within the enteroendocrine L cells located predominately in the distal small intestine and colon [36], and carbohydrates and protein are strong stimulants of these hormones within this segment of the intestinal tract [37-41]. The high fiber content of the HF-AXOS and HF-FLAX RTECs may have caused carbohydrate and protein residues to reach the distal small intestine within the first hour after consumption [39,42]. Digestion and absorption of carbohydrate and protein in the distal small intestine would then contribute to greater observed elevation of GLP-1 and PYY when the high-fiber RTECs were consumed than when the low-fiber RTECs were consumed.

In the later portion of the interval between breakfast and lunch consumption, it is likely that short-chain fatty acids (SCFA) were produced from the fermentation of the HF-AXOS and HF-FLAX high-fiber containing RTECs, and that the SCFA stimulated L cells in the distal small intestine and colon to cause GLP-1 and PYY secretion [43,44]. Our subjects reported an increase in flatulence and gas/bloating after consuming the high-fiber RTECs, indicating that fermentation was occurring and SCFA were likely being formed $[45,46]$. The high solubility and low molecular weight of the two fibers added to our high-fiber RTECs likely contributed to rapid fermentation and SCFA production [15,47]. 


\subsection{Plasma Ghrelin, Glucose, and Insulin}

Plasma ghrelin concentrations were similar after consumption of all 4 breakfast cereals and followed an expected postprandial pattern consistent with our reported VAS appetite scores. Ghrelin is strongly associated with perceived hunger [48], and we observed a time-dependent change in appetite (hunger) and ghrelin concentration. We did observe a lower plasma active ghrelin concentration at the 2 and $3 \mathrm{~h}$ time points with the high-energy control RTEC versus the low-energy control RTEC, suggesting the greater available calories in the high-energy RTEC might have delayed the return to baseline concentrations in ghrelin. The ghrelin difference observed was relatively small, and may not have been sufficient to elicit a perceived appetite change nor a change in lunch meal energy intake. These lower ghrelin concentrations with the high-energy control RTEC at $2 \mathrm{~h}$ and $3 \mathrm{~h}$ also corresponded to a higher concentration of insulin at the same time points. Blom et al. [49] demonstrated a significant negative correlation between plasma ghrelin and insulin concentrations between 30 and 180 min and concluded that insulin may contribute to ghrelin suppression.

Viscous fiber consumption has been shown to attenuate plasma glucose and insulin concentrations [50,51]. The steam pressure-cooking used to make the RTEC reduced the FLAX fiber molecular weight and viscosity of the cereal such that cereals containing FLAX and AXOS each had a relatively low viscosity [15]. This reduced the FLAX fiber's ability to attenuate plasma glucose and insulin concentrations.

\section{Conclusions}

No differences in perceived appetite, satiety, or lunch intake were observed with $15 \mathrm{~g}$ of added AXOS or FLAX fiber in a RTEC breakfast when consumed by overweight women. Collectively, these data suggest that $15 \mathrm{~g}$ of low molecular weight fiber added to RTEC did not affect perceived appetite, satiety, or energy intake at lunch $4 \mathrm{~h}$ after breakfast despite differences in satiety hormone signaling in overweight females.

\section{Acknowledgments}

This work was supported by funding from the Kellogg Company, to enable partial fulfillment of the Ph.D. degree requirements for the first author.

We thank Gary Day and Guoshen Yang for their help preparing RTEC, Toine Hulshof for advice on the study design, Valerie Kaden for her assistance managing the trials, and Arianne Schild, Kernon Gibes, and Tony Gualtieri for statistics support.

\section{Author Contributions}

David W. Lafond, Kathryn A. Greaves, Kevin C. Maki, and Dale R. Romsos conceived and designed the experiments; Kevin C. Maki and Heather J. Leidy performed the experiments; David W. Lafond, Kevin C. Maki, Heather J. Leidy, and Dale R. Romsos analyzed the data; Kevin C. Maki and Heather J. Leidy contributed reagents/materials/analysis tools; David W. Lafond, Kathryn A. Greaves, Kevin C. Maki, Heather J. Leidy, and Dale R. Romsos wrote the paper. 


\section{Conflicts of Interest}

The authors declare no conflict of interest. The Kellogg Company had no role in the design of the study, in the collection, analyses, or interpretation of data, or in the writing of the manuscript; however, it did reserve the rights to review the results prior to publishing.

\section{References}

1. Blundell, J.; Burley, V. Satiation, satiety and the action of fiber on food intake. Int. J. Obes. 1987, 11, 9-25.

2. Howarth, N.C.; Saltz, E.; Roberts, S.B. Dietary fiber and weight regulation. Nutr. Rev. 2001, 59, 129-139.

3. Pereira, M.A.; Ludwig, D.S. Dietary Fiber and Body Weight Regulation Observations and Mechanisms. Pediatr. Clin. North Am. 2001, 48, 969-980.

4. US Department of Agriculture Agricultural Research Service. What We Eat in America NHANES 2007-2008; US Department of Agriculture Agricultural Research Service: Beltsville, MD, USA, 2010.

5. Institute of Medicine of the National Academies. Dietary Reference Intakes for Energy, Carbohydrates, Fiber, Fat, Fatty Acids, Cholesterol, Protein, and Amino Acids; The National Academies Press: Washington, DC, USA, 2005.

6. Oriz, D.E.; Lafond, D.W. Physicochemical properties of wheat bran and related application challenges. In Dietary Fiber and Health; Cho, S.S., Almeida, N., Eds.; CRC Press: Boca Raton, FL, USA, 2012; pp. 369-384.

7. Liu, W.; Xie, Z.; Zhang, B.; Wang, Q.; Yao, W.; Gao, X.; Yu, L. Effects of hydroxypropylation on the functional properties of psyllium. J. Agric. Food Chem. 2010, 58, 1615-1621.

8. Swennen, K.; Courtin, C.; Lindemans, G.; Delcour, J. Large-scale production and characterization of wheat bran arabinoxylooligosaccharides. J. Sci. Food Agric. 2006, 86, 1722-1731.

9. Slavin, J.; Green, H. Fiber and Satiety. Br. Nutr. Found. Nutr. Bull. 2007, 32, 32-42.

10. Hiza, H.; Fungwe,T.; Bente, L. USDA Center for Nutrition Policy and Promotion, 2007. CNPP Publications. Available online: http://www.cnpp.usda.gov/Publications/FoodSupply/FiberFact Sheet.pdf (accessed on 2 February 2014).

11. Wanders, A.J.; Van den Borne, J.J.; de Graaf, C.; Hulshof, T.; Jonathan, M.C.; Kristensen, M.; Mars, M.; Schols, H.A.; Feskens, E.J.M. Effects of dietary fiber on subjective appetite, energy intake and body weight: A systematic review of randomized controlled trials. Obes. Rev. 2011, 12, 724-739.

12. Both-Orthman, B.R.; Rubinow, D.R.; Hoban, M.C.; Malley, J.; Grover, G.N. Menstrual cycle phase-related changes in appetite in patients with premenstrual syndrome and in control subjects. Am. J. Psychiatry 1988, 145, 628-631.

13. Davidsen, L.; Vistisen, B.; Astrup, A. Impact of the menstrual cycle on determinants of energy balance: A putative role in weight loss attempts. Int. J. Obes. 2007, 31, 1777-1785.

14. Khan, K.; Shewry, P.R. Wheat Chemistry and Technology, 4th ed.; AACC International Inc.: St. Paul, MN, USA, 2009. 
15. Lafond, D.W.; Jin, Y.; Cho, K.; Romsos, D.R. Processing affects the physicochemical properties of arabinoxylans in ready-to-eat (RTE) flaked cereal. Cereal Chem. 2015, submitted for publication.

16. Meilgaard, M.; Civille, G.V.; Carr, B.T. Sensory Evaluation Techniques; CRC Press LLC.: Boca Raton, FL, USA, 1999.

17. Flint, A.; Raben, A.; Blundell, J.; Astrup, A. Reproducibility, power, and validity of visual analogue scales in assessment of appetite sensations in single test meal studies. Int. J. Obes. Relat. Metab. Disord. 2000, 24, 38-48.

18. Buemann, B.; Toubro, S.; Astrup, A. Human Gastrointestinal Tolerance to D-Tagatose. Regul. Toxicol. Pharmacol. 1999, 29, S71-S77.

19. Shapiro, S.S.; Wilk, M.B. An analysis of variance test for normality. Biometrika 1965, 52, 591-611.

20. Brouns, F.; Bjorck, I.; Frayn, K.N.; Gibbs, A.L.; Lang, V.; Slama, G.; Wolever, T.M.S. Glycemic index methodology. Nutr. Res. Rev. 2005, 18, 145-171.

21. Sidak, Z. On probabilities of rectangles in multivariate student distributions: Their dependence on correlations. Ann. Math. Stat. 1971, 42, 169-175.

22. Freeland, K.R.; Anderson, G.H.; Wolever, T.M.S. Acute effects of dietary fiber and glycemic carbohydrate on appetite and food intake in healthy males. Appetite 2009, 52, 58-64.

23. Samra, R.A.; Anderson, G.H. Insoluble cereal fiber reduces appetite and short-term food intake and glycemic response to food consumed 75 min later by healthy men. Am. J. Clin. Nutr. 2007, 86, 972-979.

24. Hamedani, A.; Akhavan, T.; Samra, R.A.; Anderson, G.H. Reduced energy intake at breakfast is not compensated for at lunch if a high insoluble fiber cereal replaces a low fiber cereal. Am. J. Clin. Nutr. 2009, 89, 1343-1349.

25. Delargy, H.J.; Burley, V.J.; O’Sullivan, K.R.; Fletcher, R.J.; Blundell, J.E. Effects of different soluble: Insoluble fiber ratios at breakfast on $24 \mathrm{~h}$ pattern of dietary intake and satiety. Eur. J. Clin. Nutr. 1995, 49, 754-766.

26. Hlebowicz, J.; Wickenberg, J.; Fahlstro, R.B.O.; Almer, L.-O.; Darwiche, G. Effect of commercial breakfast fibre cereals compared with corn flakes on postprandial blood glucose, gastric emptying, and satiety in healthy subjects: A randomized blinded crossover trial. Nutr. J. 2007, 6, 22.

27. Delargy, H.J.; O’Sullivan, K.R.; Fletcher, R.J.; Blundell, J.E. Effects of amount and type of dietary fiber (soluble and insoluble) on short-term control of appetite. Int. J. Food Sci. Nutr. 1997, 48, 67-77.

28. Solah, V.A.; Kerr, D.A.; Adikara, C.D.; Meng, X.; Binns, C.W.; Zhu, K.; Devine, A.; Prince, R.L. Differences in satiety effects of alginate and whey protein based foods. Appetite 2010, 54, 485-491.

29. Hoad, C.L.; Rayment, P.; Spiller, R.C.; Marciani, L.; Alonso, B.; Traynor, C.; Mela, D.J.; Peters, H.P.F. In vivo imaging of intragastric gelation and its effect on satiety in humans. J. Nutr. 2004, 134, 2293-2300.

30. Rigaud, D.; Paycha, F.; Meulemans, A.; Merrouche, M.; Mignon, M. Effect of psyllium on gastric emptying, hunger feeling and food intake in nomal volunteers: A double blind study. Eur. J. Clin. Nutr. 1998, 52, 239-245.

31. Mattes, R.D.; Rothacker, D. Beverage viscosity is inversely related to postprandial hunger in humans. Physiol. Behav. 2001, 74, 551-557. 
32. Kristensen, M.; Jensen, M.G. Dietary fibers in the regulation of appetite and food intake, importance of viscosity. Appetite 2010, 56, 65-70.

33. Adam, T.C.M.; Westerterp-Plantenga, M.S. Glucagon-like peptide-1 release and statiety after a nutrient challenge in normal weight and obese subjects. Br. J. Nutr. 2005, 93, 845-851.

34. Levine, A.S.; Tallman, J.R.; Grace, M.K.; Parker, S.A.; Billington, C.J.; Levitt, M.D. Effect of breakfast cereals on short-term food intake. Am. J. Clin. Nutr. 1989, 50, 1303-1307.

35. Sorensen, L.B.; Moller, P.; Flint, A.; Martens, M.; Raben, A. Effect of sensory perceptions of foods on appetite and food intake: A review of studies on humans. Int. J. Obes. Relat. Metab. Disord. 2003, 10, 1152-1166.

36. Habib, A.M.; Richards, P.; Rogers, G.J.; Reimann, F.; Gribble, F.M. Co-localisation and secretion of gluagon-like peptide 1 and peptide YY from primary cultured human L cells. Diabetologia 2013, 56, 1413-1416.

37. Gribble, F.M.; Williams, L.; Simpson, A.K.; Reiman, F. A novel glucose sensing mechanism contributing to glucagon-like peptide-1 secretion from the GLUTag cell line. Diabetes 2003, 52, 1147-1154.

38. Little, T.J.; Doran, S.; Meyer, J.H.; Smout, A.J.P.M.; O’Donovan, D.G.; Wu, K.-L.; Jones, K.L.; Wishart, J.; Rayner, C.K.; Horowitz, M.; Feinle-Bisset, C. The release of GLP-1 and ghrelin, but not GIP and CCK, by glucose is dependent upon the length of small intestine exposed. Am. J. Physiol. Endocrinol. Metab. 2006, 291, E647-E655.

39. Van der Klaauw, A.A.; Keogh, J.M.; Henning, E.; Trowse, V.M.; Dhillo, W.S.; Ghatei, M.A.; Farooqi, I.S. High protein intake stimulates postprandial GLP-1 and PYY release. Obesity 2013, 21, 1602-1607.

40. Lomenick, J.P.; Melguizo, M.S.; Mitchell, S.L.; Summar, M.L.; Anderson, J.W. Effects of meals high in carbohydrate, protein, and fat on ghrelin and peptide YY secretion in prepubertal children. J. Clin. Endocrinol. Metab. 2009, 94, 4463-4471.

41. Batterham, R.L.; Heffron, H.; Kapoor, S.; Chivers, J.E.; Chandarana, K.; Herzog, H.; Le Roux, C.W.; Thomas, E.L.; Bell, J.D.; Withers, D.J. Critical role for peptide YY in protein mediated satiation and body weight regulation. Cell Metab. 2006, 4, 223-233.

42. Ou, S.; Kwok, K.; Li, Y.; Fu, L. In vitro study of possible role of dietary fiber in lowering postprandial serum glucose. J. Agric. Food Chem. 2001, 49, 1026-1029.

43. Tolhurst, G.; Heffron, H.; Lam, Y.S.; Parker, H.E.; Habib, A.M.; Diakogiannaki, E.; Cameron, J.; Grosse, J.; Reimann, F.; Gribble, F.M. Short-chain fatty acids stimulate glucagon-like peptide-1 secretion via the G-protein-coupled receptor FFAR2. Diabetes 2012, 61, 364-371.

44. Cani, P.D.; Lecourt, E.; Dewulf, E.M.; Sohet, F.M.; Pachikian, B.D.; Naslain, D.; De Backer, F.; Neyrinck, A.M.; Delzenne, N.M. Gut microbiota fermentation of prebiotics increases satietogenic and incretin gut peptide production with consequences for appetite sensation and glucose response after a meal. Am. J. Clin. Nutr. 2009, 90, 1236-1243.

45. Francois, I.E.J.A.; Lescroat, O.; Veraverbeke, W.S.; Marzorati, M.; Possemiers, S.; Evenepoel, P.; Hamer, H.; Houben, E.; Windey, K.; Welling, G.W.; et al. Effects on wheat bran extract containing arabinoxylan oligosaccharides on gastrointestinal health parameters in healthy adult human volunteers; a double-blind, randomized, placebo-controlled, cross-over trial. Br. J. Nutr. 2012, 108, 2229-2242. 
46. Neyrinck, A.M.; Van Hee, V.F.; De Backer, F.; Toussaint, O.; Cani, P.D.; Delzenne, N.M. Wheat derived arabinoxylan oligosaccharides with prebiotic effect increase satietogenic gut peptides and reduce metabolic endotoxemia in diet induced obese mice. Nutr. Diabetes 2012, 2, 1-9.

47. Slavin, J. Fiber and prebiotics: Mechanisms and health benefits. Nutrients 2013, 5, 1417-1435.

48. Delzenne, N.; Blundell, J.; Brouns, F.; Cunningham, K.; De Graaf, K.; Erkner, A.; Lluch, A.; Mars, M.; Peters, H.P.F.; Westerterp-Plantenga, M. Gastrointestinal targets of appetite regulation in humans. Obes. Rev. 2010, 11, 234-250.

49. Blom, W.A.M.; Stafleu, A.; de Graaf, C.; Kok, F.J.; Schaafsma, G.; Hendriks, H.F.J. Ghrelin response to carbohydrate-enriched breakfast is related to insulin. Am. J. Clin. Nutr. 2005, 81, 367-375.

50. Regand, A.; Tosh, S.M.; Wolever, T.M.S.; Wood, P.J. Physicochemical properties of $\beta$-glucan in differently processed oat foods influence glycemic response. J. Agric. Food Chem. 2009, 57, 8831-8838.

51. Dikeman, C.L.; Fahey, G.C. Viscosity as related to dietary fiber: A review. Crit. Rev. Food Sci. Nutr. 2006, 46, 649-663.

(C) 2015 by the authors; licensee MDPI, Basel, Switzerland. This article is an open access article distributed under the terms and conditions of the Creative Commons Attribution license (http://creativecommons.org/licenses/by/4.0/). 